\title{
Persistent Yersinia enterocolitica infection in three rat strains
}

\author{
J. H. A. J . Curfs*, J . F. G. M. Meis, H. A. L. Van der Lee, J . A. Mulder, \\ W. A. G. Kraak and J . A. A. Hoogkamp-Korstanje
}

Department of Medical Microbiology, University Hospital Nijmegen, P.O. Box 9101, 6500 HB Nijmegen, The Netherlands

(Received October 3, 1994; accepted in revised form J une 28, 1995)

Curfs, J . H. A. J . (Department of Medical Microbiology, University Hospital Nijmegen, P.O. Box 9101, 6500 HB Nijmegen, The Netherlands), J . F. G. M. Meis, H. A. L. van der Lee, J. A. Mulder, W. A. G. Kraak and J. A. A. Hoogkamp-Korstanje. Persistent Yersinia enterocolitica infection in three rat strains. Microbial Pathogenesis 1995; 19: 57-63.

Yersinia enterocolitica may persist for prolonged periods of time in humans sometimes resulting in the development of reactive arthritis. To elucidate factors predisposing for persistence we developed animal models. In Lewis and Fischer rats, viable bacteria could be demonstrated for prolonged time and abscesses could be found in the liver, spleen and lungs. Splenic abscesses were observed for more than 20 weeks. Yersinia enterocolitica persisted in Lewis and Fischer rats, but only Lewis rats developed reactive arthritis. In Brown Norway rats abscesses developed early during infection but in contrast to the other strains disappeared after 3 weeks. Culture of homogenized abscess-containing tissue of all three rat strains yielded Yersiniae. Immunofluorescence studies of the abscesses showed diffuse staining inside the abscesses only, indicating the presence of Yersinia enterocolitica antigen. Brown Norway rats, in contrast to Lewis and Fischer rats, developed a different serological reaction pattern against Yersinia enterocolitica antigens and this correlated with the disappearance of the abscesses.

(c) 1995 Academic Press Limited

Key words: Yersinia; Rats; Arthritis; Persistence; Yops; Antibody.

\section{Introduction}

Gastrointestinal infection with Yersinia enterocolitica is self-limiting in most cases because the host's inflammatory response is usually able to eliminate the bacteria. In some cases infection with $Y$. enterocolitica as well as infection with a variety of micro-organisms, such as Salmonella and Chlamydia ${ }^{1,2}$ may lead to the development of a persistent infection with or without the ensuing development of sequellae such as reactive arthritis. It is unknown why some people develop such a chronic infection whereas others do not. Persistence of the causative organism or bacterial antigens somewhere within the body, thereby maintaining a stimulus for a prolonged immune response and inflammation, might explain the persistence of $Y$. enterocolitica-specific IgA antibodies in patients with chronic infection or $Y$. enterocolitica-associated reactive arthritis. ${ }^{3,4} \mathrm{~A}$ prerequisite for the development of reactive arthritis seems to

* Author to whom correspondence should be addressed. 
be the persistent presence of bacteria or bacterial antigens in the body. ${ }^{4-7}$ Typically, no bacteria can be cultured from affected joints which separates reactive arthritis from septic arthritis. However, bacterial antigens (immune-complexes) have been demonstrated. ${ }^{5,7}$ In addition, the majority of patients developing reactive arthritis as well as other spondylarthropathies have the MHC class I antigen typing of HLA-B27..$^{-11}$

Persistent $Y$. infection in animal models followed by the development of reactive arthritis has been described previously. Lew is and spontaneously hypertensive (SHR) rats ${ }^{6,12-15}$ as well as several strains of mice ${ }^{16}$ develop arthritis after infection with $Y$. enterocolitica. Here we describe and compare a new rat model that might provide useful information to study the pathogenesis of persistent $Y$. enterocolitica infections and ensuing sequellae.

\section{Results}

\section{Characteristics of Y.enterocolitica infection in different rat strains}

Lewis rats. Two weeks after inoculation with $Y$. enterocolitica multiple macroscopic abscesses were found in the spleen of all animals. In some of the animals abscesses were also found in the liver (40\%) and lungs (13.3\%). Yersiniae were recovered from all abscesses. Liver and lungs without macroscopic visible abscesses (i.e. day 7 after infection) also yielded positive cultures. Internal organs from uninfected rats as well as blood and Peyer's patches from infected rats did not contain any viable Yersiniae. Splenic abscesses persisted for more than 138 days and reached maximal sizes of $3.5 \mathrm{~cm}$ diameter. Throughout this period Yersiniae could be cultured from the abscesses. Abscesses in liver and lungs disappeared 5 to 6 weeks after infection and these organs became culture negative.

Microscopic examination of splenic tissue revealed abscesses encapsulated with a thick fibroblastic layer (Fig. 1A). Infiltration of granulocytes was a prominent feature

(a)

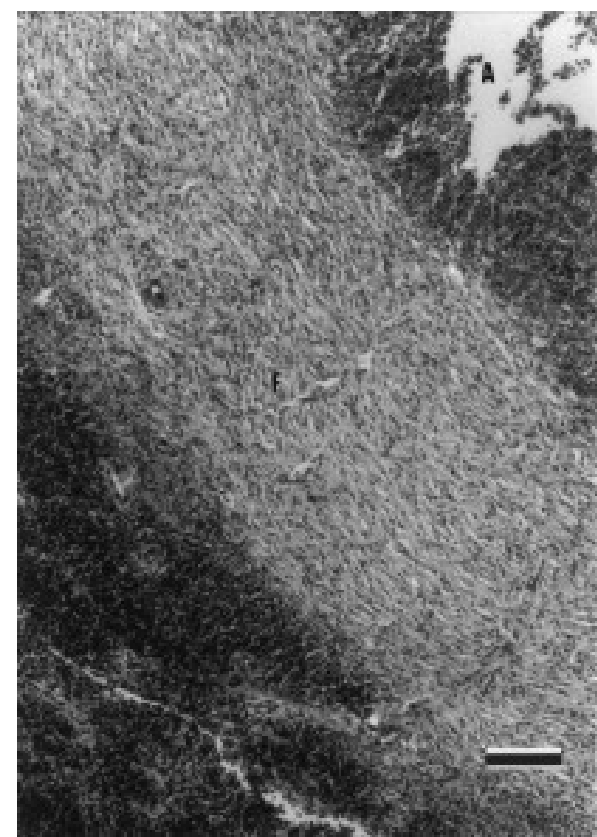

(b)

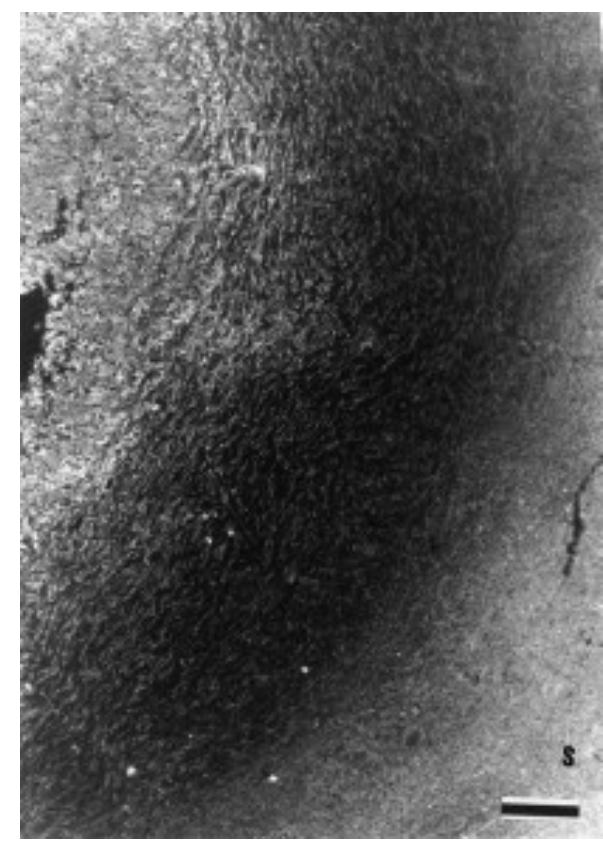

Fig. 1. Spleen of Lewis rat with large abscess 5 weeks after i.v. infection with $10^{4}$ cfu Yersinia enterocolitica $0: 8 W A$ (5 $\mu \mathrm{m}$ section). (a) hematoxylin-eosin stain; (b) immunofluorescence. $a=a b s c e s s, f=$ fibroblast capsule, $\mathrm{s}=$ normal splenic tissue. Bar represents $25 \mu \mathrm{m}$. 


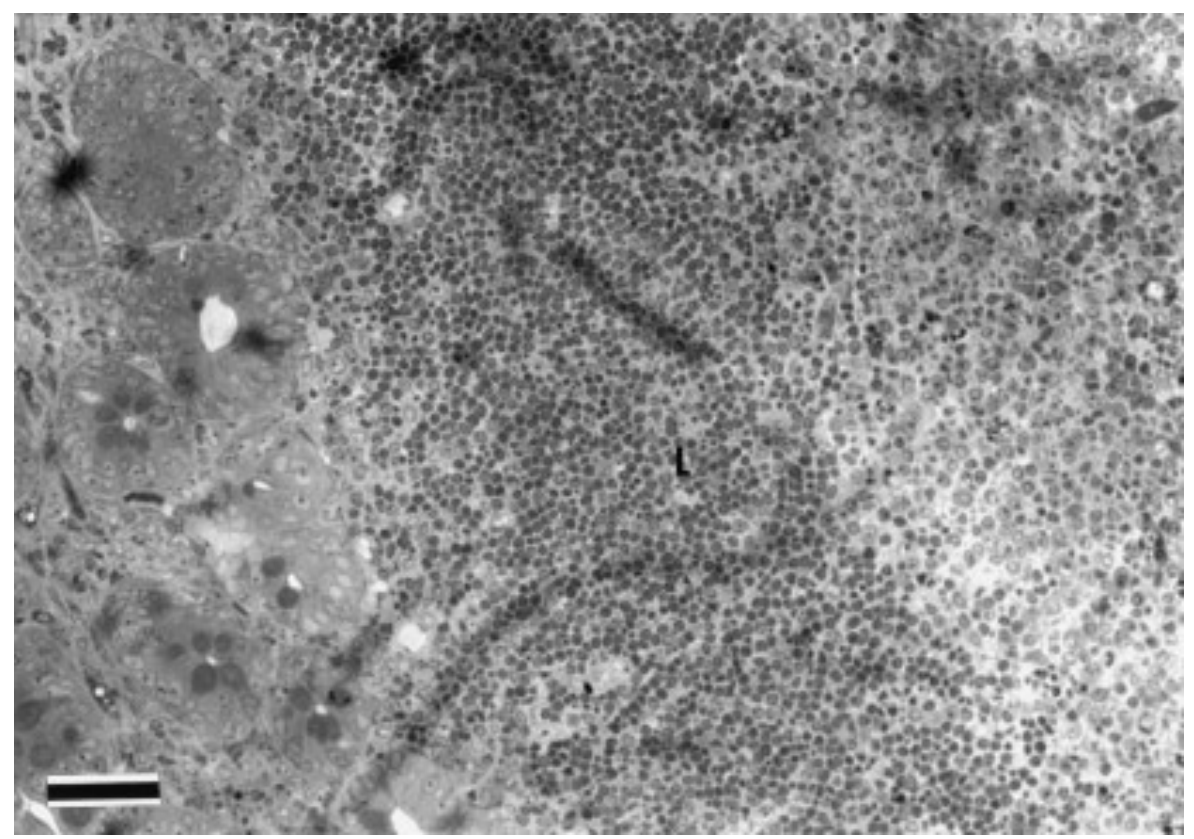

Fig. 2. Peyer's Patch of Lewis rat 2 weeks after i.v. infection with $10^{4} \mathrm{cfu}$ Yersinia enterocolitica $0: 8 W A$. Toluidine Blue stain ( $1 \mu \mathrm{m}$ section). Notice proliferation of lymphatic tissue (L). Bar represents $25 \mu \mathrm{m}$.

of the abscesses, however intact bacteria could not be distinguished. Immunohistological staining of the splenic tissue revealed strong, diffuse fluorescence within the abscesses (Fig. 1B). No fluorescence was observed in the fibroblastic layer or in the unaffected splenic tissue, indicating that bacteria or bacterial antigens are located inside the abscesses. As in the spleen, immunofluorescence of liver sections was confined to abscesses (results not shown). Although no bacteria could be demonstrated within the Peyer's patches, histological examination revealed proliferation of lymphatic tissue (Fig. 2).

Serum samples taken at sequential timepoints after infection showed the development of an antibody response against five outer proteins (Yops) of Yersinia (Yops $\mathrm{O}, \mathrm{M}, \mathrm{H}, \mathrm{D}$ and $\mathrm{N}$ ) within two weeks after inoculation of bacteria (Fig. 3A). These antibody levels persisted for more than 138 days.

Three weeks after injection of bacteria 12 out of 15 Lewis rats had developed arthritis. Swelling of a single, or in two cases both hind paws could be observed. In the fifth week after infection the swelling began to subside and 2 weeks later no distinction could be made between rats with and without an episode of arthritis. Before, during and after the episode of arthritis no bacteria could be cultured from the affected joints.

Fischer rats. After inoculation of bacteria the same pathology could be observed in Fischer rats as in Lewis rats with the exception of the development of reactive arthritis which was absent in Fischer rats. Macroscopic abscesses were found in the spleen of all animals, and sometimes in liver (33.3\%) and lungs (20\%). Antibody reactivity in Fischer rats did not differ from that observed in Lewis rats (Fig. 3B).

Brown Norway rats. In Brown Norway rats abscesses developed in spleen (100\%), liver (20.6\%) and lungs (13.3\%) and bacteria could be cultured from these organs. Histology showed the same characteristics as described for Lewis and Fischer rats. 


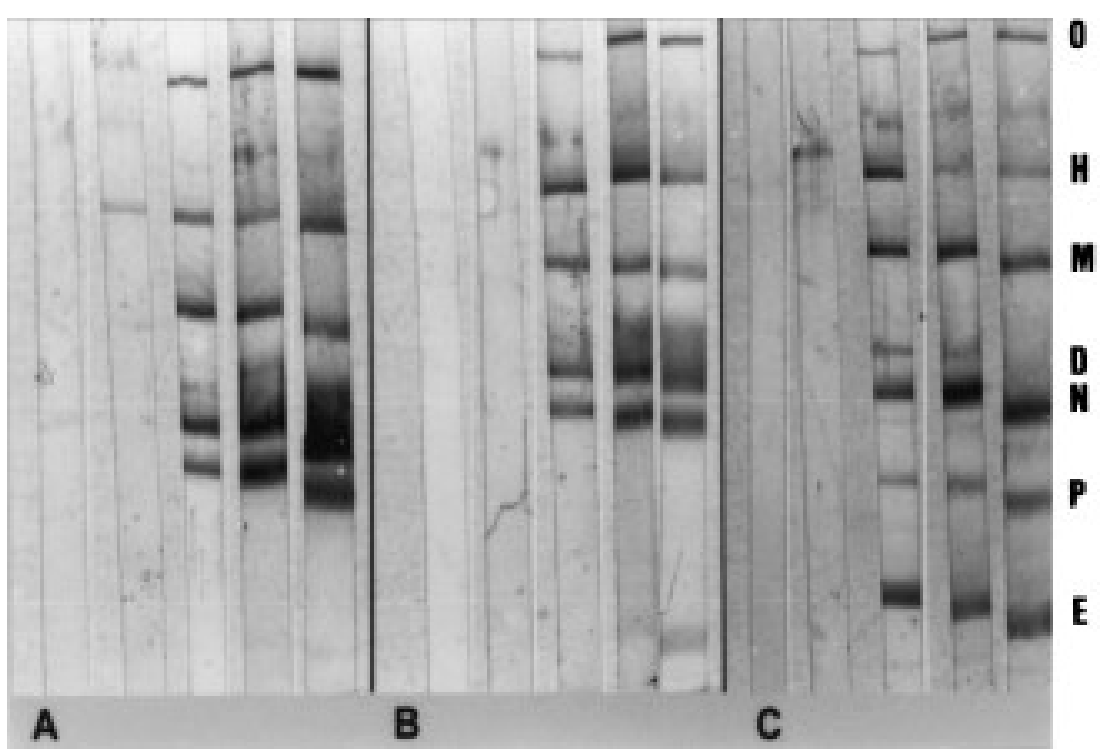

Fig. 3. Immunoblot of serum from individual rats after infection with Yersinia enterocolitica $0: 8 W A$. From left to right lanes indicate serum samples taken on day $0,7,14,22$ and 35 after infection. $A=$ Lewis rats, $B=$ Fischer rats, $C=$ Brown Norway rats. $M$ ol. weight Yops: $Y o p O=84$ kD, $Y o p H=51$ kD, YopM $=48$ kD, YopD $=37 \mathrm{kD}, \mathrm{YopN}=35 \mathrm{kD}, \mathrm{YopP}=30 \mathrm{kD}$ and $\mathrm{YopE}=25 \mathrm{kD}$.

However, after 21 days abscesses decreased in size and all organs became culture negative.

All Brown Norway rats produced antibodies against $Y$ ops $\mathrm{O}, \mathrm{M}, \mathrm{H}, \mathrm{D}, \mathrm{N}$ and against two additional Yops, YopP and YopE (Fig. 3C), and this response became prominent at the time that abscesses began to decrease in size. During the course of the infection humoral reactivity against Yops $\mathrm{O}, \mathrm{M}, \mathrm{H}, \mathrm{D}$ and $\mathrm{N}$ ultimately decreases as indicated by the fading of the immunoblot pattern against these Yops. No reactive arthritis developed in Brown Norway rats.

\section{Discussion}

Humans infected with $Y$. enterocolitica serotypes $0: 3,0: 8$ or $0: 9$ sometimes develop a chronic infection with persistent levels of antibodies (IgA subclass) against Yersinia enterocolitica antigens. ${ }^{4}$ Persistent infection with either of the three serotypes may be accompanied by the development of sequellae such as reactive arthritis. ${ }^{17}$ Studies in rat models have also shown that infection with Y. enterocolitica may lead to a chronic infection with persistence of the bacteria somewhere within the affected host. ${ }^{12}$ Additional evidence for the prolonged presence of bacteria in infected rats is presented here. After infection, all three rat strains developed splenic abscesses from which Yersiniae could be cultured. Furthermore, strong immunofluorescence was found inside the abscesses whereas unaffected tissue lacked any label. This indicates that viable bacteria are located in the abscesses rather than in normal splenic tissue. Although no bacteria could be demonstrated inside Peyer's Patches, proliferation of the lymphatic tissue indicates an inflammatory response.

Yops are strong antigens and elicit a specific $\lg \mathrm{M}$-, IgA- and IgG-antibody response in orally infected rabbits, ${ }^{18}$ in humans suffering from Yersinia infection ${ }^{19}$ and in rats. ${ }^{6}$ We find persistent antibody levels directed against several Yops in infected Lewis and Fischer rats in contrast to Brown Norway rats where antibody reactivity began 
to fade from the fifth week of infection and onwards. This might reflect persistence of bacteria inside the infected host since the vanishing of Yop-specific antibodies in sera from Brown Norway rats coincided with the disappearance of abscesses and bacteria. Development of a humoral response against YopP and YopE was found exclusively in Brown Norway rats and was associated with disappearance of splenic abscesses. Failure to mount an anti-YopP and/or anti-YopE response by Lewis and Fischer rats may therefore be correlated with persistence of bacteria suggesting that YopP and/or YopE may be suitable target-antigens for effective elimination of the bacteria by the immune system. Furthermore, antibody reactivity againstYopE might render the bacteria susceptible to phagocytosis since YopE is supposed to be involved in resistance against phagocytosis through depolymerization of the cellular actin network. ${ }^{20}$

Differences in kinetics of antibody production against Yops have been shown by Gaede et al. ${ }^{6}$ They found a weaker and delayed antibody response against Yops in Fischer rats compared to Lewis rats after infection with $\mathrm{Y}$. enterocolitica $\mathrm{O}: 8 \mathrm{WA}$. In their study Lewis rats developed reactive arthritis whereas Fischer rats did not. Also in our experiments Lewis rats developed reactive arthritis in contrast to Fischer rats but no evidence for a delayed antibody response was found in Fischer rats which make it unlikely that a relatively early antibody response may explain the development of reactive arthritis in Lewis rats.

As described by Hill and $\mathrm{Yu}^{12}$ the development of reactive arthritis after infection of rats with $\mathrm{Y}$. enterocolitica $\mathrm{O}: 8 \mathrm{WA}$ is closely linked to the prolonged presence of the causing organism somewhere in the affected host. In their experiments Lewis rats developed a persistent infection in the spleen followed by arthritis whereas Fischer rats cleared the bacteria from spleen and liver within 2-3 weeks and did not develop arthritis. They indicated that the persistence of bacteria in Lewis rats might be the reason for the development of reactive arthritis. In addition, experiments with SHR rats ${ }^{15}$ suggested that the development of arthritis depended on persistence of the bacteria in the affected host. However, in our experiments Lewis as well as Fischer rats developed a persistent infection but only Lewis rats developed reactive arthritis. The reason why in our experiments Lewis rats developed reactive arthritis and Fischer rats did not still remains unclear since Yersinia persisted in both rat strains. As described in human reactive arthritis the genetic background (MHC type) may play a role. ${ }^{9}$ However, SHR (Spontaneously Hypertensive Rats) and WKY rats (Wistar Kyoto, the normotensive strain of SHR) that share the same histocompatibility antigens, differ in their susceptibility to $\mathrm{Y}$. enterocolitica-induced arthritis. ${ }^{13}$ Furthermore, the microbial status of the host may play a role as described by GripenbergLerche and Toivanen. ${ }^{21,22}$ This indicates that the development of reactive arthritis following a persistent infection with Y. enterocolitica may depend on the immunestatus rather than on the MHC type which may explain the difference between our results and those observed by others. ${ }^{6,12}$

\section{Materials and methods}

Experimental animals. Male rats weighing at least $300 \mathrm{~g}$ were used in all experiments. Lewis and Fischer rats were obtained from colonies maintained at the central Animal Laboratory, University Hospital Nijmegen and Brown Norway rats were obtained from TNO, Rijswijk, The Netherlands. Rats were housed in plastic cages under specific pathogen free conditions and received water and food ad libitum. For persistence studies five rats of each strain were used and the experiment was performed three times. 
Bacteria. Yersinia enterocolitica serotype 0 : 8WA (ATCC 9610) was used in all experiments. This strain contains the virulence plasmid. Bacteria were cultured overnight in Brain Heart Infusion broth $(\mathrm{BHI})$ at $26^{\circ} \mathrm{C}$. The concentration of inoculated viable bacterial cells was estimated by colony counts on Columbia blood agar plates.

Infection. For all experiments animals were infected intravenously with approximately $10^{4}$ cfu in $0.2 \mathrm{ml} 0.9 \%$ saline.

Individual animals from each rat strain were killed 7, 14, 22, 40 and 138 days after inoculation with Y. enterocolitica. Spleen, liver, lungs and Peyer's patches were examined for the presence of macroscopic lesions and assayed for live bacteria. Abscess-containing tissue samples were prepared for histology.

For serological studies five rats of each strain were inoculated with $\mathrm{Y}$. enterocolitica and serum samples were taken at serial intervals. In three independent experiments samples were taken on days $0,7,14,22$ and 35 after inoculation and were examined for antibody reactivity against Yops.

Antisenum. New Zealand White rabbits were obtained from colonies maintained at the Central Animal Laboratory, University Hospital Nijmegen. Rabbits were immunized with Yersinia enterocolitica O : 8WA (ATCC 9610) as described by Hanski et al. ${ }^{22}$ Briefly, bacteria were grown overnight at $26^{\circ} \mathrm{C}$ in $\mathrm{BHI}$ broth containing $20 \mathrm{~mm}$ calciumchloride and $20 \mathrm{~mm}$ sodiumoxalate. Formaldehyde fixed bacteria ( $10^{9} \mathrm{cfu}$ in $1 \mathrm{ml}$ saline) mixed with Complete Freund's Adjuvants (1:1) were injected intracutaneously on 10 locations on the back of the rabbits followed by booster injections with $10^{5} \mathrm{cfu}$ in $1 \mathrm{ml}$ saline mixed with Incomplete Freund's Adjuvants (1:1) 2 and 4 weeks later. Eight days after the second booster the animals were bled by cardiac puncture and the serum was collected and stored at $-20^{\circ} \mathrm{C}$.

Bacterial culture. Culture of bacteria from homogenized tissue samples of spleen, liver, lungs and Peyer's patches was carried out on Columbia blood agar plates and selective CIN plates (Cefsulodin Irgasan Novobiocin, Oxoid). API 20E or 50SH was used to identify Yersinia enterocolitica.

Microscopy. Routine histological procedures were used for preparation of tissue samples for light microscopy. For immunofluorescence, $5 \mu \mathrm{m}$ cryosections were allowed to dry overnight and were then incubated with rabbit-anti-Yersinia enterocolitica serum diluted $1: 250$ in $0.05 \%$ Tween-20 in PBS. After washing twice with PBS/Tween, the sections were incubated with swine-anti-rabbit antibodies coupled to FITC (Dako A/S, Glostrup, Denmark; dilution 1: 50 in PBS/0.05\% Tween-20). After washing twice, sections were embedded in Gurr Aquamountant (BDH Laboratory Suppliers, Poole, U. K.) and examined in a fluorescence microscope.

Serology. SDS-PAGE/Immunoblotting technique ${ }^{24}$ was used to determine the presence of antibodies directed against plasmid encoded Yops. Briefly, Yersinia was grown overnight at $26^{\circ} \mathrm{C}$ in $\mathrm{BHI}$ broth containing $20 \mathrm{~mm} \mathrm{MgCl}_{2}$ and $20 \mathrm{~mm}$ EGTA followed by $3 \mathrm{~h}$ incubation at $37^{\circ} \mathrm{C}$. After centrifugation released proteins from the supernatant were precipitated with $\left(\mathrm{NH}_{4}\right)_{2} \mathrm{SO}_{4}$ and separated by SDS-PAGE followed by Western blotting. Specific antibodies against Yops were detected by a subsequent incubation of blot strips with rat sera (dilution $1: 250)$ followed by rabbit-anti-Ig coupled to alkaline phosphatase (1:500).

\section{References}

1. Sieper J, Braun J , Brandt J et al. Pathogenic role of Chlamydia, Yersinia and Borrelia in undifferentiated oligoarthritis. J. Rheumatol 1992; 19: 1236-42.

2. Toivanen A, Granfors K, Lahesmaa-Rantala R, Leino R, Stahlberg T, Vuento R. Pathogenesis of Yersinia-triggered reactive arthritis: immunological, microbiological and clinical aspects. Immunol Rev 1985; 86: 47-70.

3. Granfors K, Viljanen M, Tiilikainen A, Toivanen A. Persistence of IgM, IgG, and IgA antibodies to Yersinia in Yersinia-arthritis. J Infect Dis 1980; 141: 424-9.

4. Hoogkamp-Korstanje J AA, de Koning J , Heesemann J . Persistence of Yersinia enterocolitica in man. Infection 1988; 16: 81-5. 
5. Granfors $\mathrm{K}$, J alkanen $\mathrm{S}$, von Essen $\mathrm{R}$ et al. Yersinia antigens in synovial-fluid cells from patients with reactive arthritis. N Engl J Med 1989; 26: 216-21.

6. Gaede K, Mack D, Heesemann J. Experimental Yersinia enterocolitica infection in rats: analysis of the immune response to plasmid-encoded antigens of arthritis-susceptible Lewis rats and arthritisresistant Fischer rats. Med Microbiol Immunol 1992; 181: 165-72.

7. Toivanen A, Lahesmaa-Rantala R, Stahlberg TH, Merilahti-Palo R, Granfors K. Do bacterial antigens persist in reactive arthritis? Clin Exp Rheumatol 1987; 5: 25-7.

8. Aho $K$, Ahvonen $P$, Lassus A, Sievers K, Tiilikainen A. HLA-B27 in reactive arthritis and Reiter's diseases. Arthr Rheum 1974; 17: 521-6.

9. Benjamin RJ , Parham P. HLA-B27 and ankylosing spondylitis. Immunol Today 1990; 11: 137-42.

10. Brewerton DA, Caffrey M, Nicholls A, Walters D, Oates J K, J ames DCO. Reiter's disease and HLA-B27. Lancet 1973; ii: 996-8.

11. Kingsley G, Sieper J . Second international workshop on reactive arthritis. Clin Exp Rheumatol. 1993; 11: 229-37.

12. Hill J L, Yong Z, Laheji K, Kono DH, Yu DTY. Experimental animal models of Yersinia infection and Yersinia-induced arthritis. Contrib Microbiol Immunol 1987; 9: 228-32.

13. Toivanen A, Merilahti-Palo R, Gripenberg C, Lahesmaa-Rantala R, Soderstrom K-O, J aakkola U-M. Yersinia-associated arthritis in the rat: experimental model for human reactive arthritis? Acta Pathol Microbiol Immunol Scand 1986; 94: 261-9.

14. Tong Z, Hill J L, Hirofuji T, Mander M, Yu DTY. An experimental mouse model of Yersinia-induced reactive arthritis. Microb Pathogen 1988; 4: 306-10.

15. Merilahti-Palo R, Gripenberg-Lerche C, Soderstrom K-O, Toivanen P. Long term follow up of SHR rats with experimental Yersinia-associated arthritis. Ann Rheum Dis 1992; 51: 91-6.

16. De los Toyos J R, Vazquez J, Sampedro A, Hardisson C. Yersinia enterocolitica serotype 0:3 is arthritogenic for mice. Microb Pathog 1990; 8: 363-70.

17. Hoogkamp-Korstanje J AA, de Koning J, Samson J P. Incidence of human infection with Yersinia enterocolitica O3, O8, O9 and the use of indirect immunofluoresence. J Infect Dis 1986; 153: 138-9.

18. Heesemann J, Eggers C, Schröder J . Serological diagnosis of yersiniosis by immunoblot technique using virulence-associated antigens of enteropathogenic Yersinia. Contrib Microbiol Immunol 1987; 9: 285-9.

19. Heeseman J, Schröder J, Ulrich M. Analysis off the class-specific immune response to Yersinia enterocolitica virulence-associated antigens in oro-gastrically infected rabbits. Microb Pathog 1988; 5: 437-47.

20. Rosqvist R, Forsberg A, Wolf-Watz H. Intracellular targeting of the Yersinia YopE cytotoxin in mammalian cells induces actin microfilament disruption. Infect Immun 1991; 59: 4562-9.

21. Gripenberg-Lerche C, Toivanen P. Yersinia-associated arthritis in SHR rats: effect of the microbial status of the host. Ann Rheum Dis 1993; 52: 223-8.

22. Gripenberg-Lerche $C$, Toivanen P. Variability in the induction of experimental arthritis: Yersiniaassociated arthritis in Lewis rats. Scand J Rheumatol 1994; 23: 124-7.

23. Hanski C, Kutschka U, Schmoranzer HP et al. Immunohistochemical and electron microscopic study of interaction of Yersinia enterocolitica serotype $0: 8$ with intestinal mucosa during experimental enteritis. Infect Immun 1989; 57: 673-8.

24. Heesemann J , Gross U, Schmidt N, Laufs R. Immunochemical analysis of plasmid-encoded proteins released by enteropathogenic Yersinia sp. grown in calcium-deficient media. Infect Immun 1986; 54 561-7. 